\title{
Design of a decision support system for predicting the progression of Alzheimer's Disease.
}

Manon Ansart ${ }^{1,2}$, Igor Koval ${ }^{1,2}$, Anne Bertrand ${ }^{2,3}$, Didier Dormont ${ }^{2,3}$, Stanley Durrleman ${ }^{1,2}$

\author{
${ }^{1}$ Sorbonne Université, UPMC Univ Paris 06, Inserm, CNRS, Institut du cerveau et la \\ moelle (ICM) - Hôpital Pitié-Salpêtrière, 75013 Paris, France \\ ${ }^{2}$ Inria Paris, ARAMIS project-team, 75013 Paris, France \\ ${ }^{3}$ Sorbonne Université, UPMC Univ Paris 06, Inserm, CNRS, Institut du cerveau et la \\ moelle (ICM), APHP - Hôpital Pitié-Salpêtrière, 75013 Paris, France
}

\begin{abstract}
Background: Knowing who will develop Alzheimer's disease (AD) and when is a crucial health issue, that could help treat subjects earlier, using new treatments targeting the earliest stages. We have participated in TADPOLE, a competition based on ADNI data aiming at developing an automatic prognosis system. We propose a method for predicting the time to conversion of $\mathrm{MCI}$ subjects to $\mathrm{AD}$ and a framework to evaluate such predictions.
\end{abstract}

Method: We decompose the prediction into two steps: first, we monthly predict future values of the cognitive scores of each subject; second, we use them to automatically predict the corresponding disease status, using a Support Vector Machine. For the first step, we compare two approaches: the univariate and the multivariate approach. In the univariate approach, cognitive scores are predicted by linear regression with respect to time for each subject. In the multivariate approach, sociodemographic, cognitive and MRI features of the last visit are used to predict the future cognitive scores. This approach is automatically learned using all visits of training subjects.

We evaluate our methods by splitting longitudinal data into training and test subjects. Each test subject is split into observed visits and a future visit, separated by different time periods (mostly 1 to 4 years in the challenge).

We want to verify three hypotheses: the multivariate approach outperforms the univariate approach; separating the process into two parts perform better than directly using a classification algorithm; the time to prediction is an important parameter that has to be taken into account.

Results: The univariate approach $(\mathrm{AUC}=82.6 \% \pm 3.0)$ performs significantly worse $(\mathrm{p}=0.0001)$ than the multivariate approach $(\mathrm{AUC}=85.5 \% \pm 2.5)$. Both perform significantly better $(\mathrm{p}<0.0001)$ than a simple classification $(\mathrm{AUC}=59.2 \% \pm 6.2)$. Using a time to prediction of 1 to 8 years, as in the challenge leaderboard, lowers the performance (AUC $=79.3 \%$ for the multivariate approach). 
Conclusion: We propose a method to predict if and when MCI subjects convert to AD. We show that a careful design of the prediction method and evaluation framework allows to outperform straightforward approaches. The method could be extended to target even earlier stages of the disease, by predicting the conversion of healthy subjects. 